\title{
Angioedema without urticaria caused by oral acitretin
}

\author{
Efstathia Pasmatzi ${ }^{1 凶}$, Alexandra Monastirli1,2, George Badavanis ${ }^{1}$, Dionysios Tsambaos ${ }^{1,2}$
}

\begin{abstract}
The efficacy of oral acitretin in the systemic treatment of severe and recalcitrant dermatoses has been established in a large number of clinical trials. Its mucocutaneous and ocular adverse reactions are common or relatively common, whereas systemic side effects are either uncommon or rare and include teratogenesis, hyperlipidemia, hepatotoxicity, intracranial hypertension, myopathy, and peripheral neuropathy. Angioedema is a term used to describe an acute, solitary or multiple, circumscribed, and non-pitting mucocutaneous swelling that affects the dermis and the subcutaneous tissue, lasts 24 to 72 hours, and may become potentially life-threatening when the pharynx and/or the larynx are involved. We report here the case of a 51-year old female psoriatic patient with angioedema (without urticaria) due to oral acitretin, confirmed by a positive oral provocation test. To the best of our knowledge, this is the second description of such an adverse reaction to acitretin.
\end{abstract}

Keywords: urticaria, angioedema, acitretin

Received: 8 April 2017 | Returned for modification: 5 May 2017 | Accepted: 18 May 2017

\section{Introduction}

In the last three decades, the dramatic efficacy of oral acitretin (representative of the second retinoid generation) in the systemic treatment of severe and recalcitrant keratinization disorders has been established in a large number of clinical trials. Its mucocutaneous and ocular adverse reactions are common or relatively common, whereas systemic side effects are either uncommon or rare and include teratogenesis, hyperlipidemia, hepatotoxicity, intracranial hypertension, myopathy, and peripheral neuropathy $(1,2)$.

Angioedema is an acute, solitary or multiple, circumscribed, non-pitting mucocutaneous swelling (due to a temporary enhancement of endothelial permeability resulting in extravasation of plasma in the dermis and the subcutaneous tissue) that lasts 24 to 72 hours (3). It may also target the gastrointestinal and respiratory tracts and become potentially life-threatening when the pharynx and/or the larynx are involved (4). Angioedema can be classified into three forms: a) an idiopathic form with unknown pathogenetic mechanisms, b) an extrinsic factor-induced form (due to histamine [IgE- and non IgE- triggered] or kinin-mediated pathophysiology), and c) a hereditary/acquired form associated with C1 esterase inhibitor deficiency or dysfunction (5).

Angioedema is a multi-etiological condition that can be induced by genetic, infectious, and physical factors, autoimmune disorders, foods, vaccines, insect bites, stings, radiocontrast media, latex, and drugs. An ever-increasing number of drugs can induce angioedema through either immunological or non-immunological mechanisms $(5,6)$. We report here the case of a 51-year-old female patient with chronic plaque psoriasis, which presented angioedema 2 days after the onset of oral acitretin treatment.

\section{Case report}

A 51-year-old Caucasian female presented with generalized chronic plaque psoriasis, which she had had for 2 years. She had been treated with various topical steroid-containing regimens but experienced frequent relapses upon discontinuation of the treatment.
The results of routine laboratory investigations were unremarkable. Thus, oral treatment with $0.8 \mathrm{mg} / \mathrm{kg} /$ day acitretin (NeoTigason, Actavis, Hafnarfjörður, Iceland) was initiated. Two days after the start of treatment, she developed a diffuse, skin-colored, non-itchy, and slightly painful swelling in the facial skin, which was more prominent in the periorbital areas and in the lips, but no wheals could be observed. Furthermore, there was no involvement of the larynx, pharynx, or any other part of her body. In the last 2 months preceding acitretin treatment, the patient had received no medications and her history and that of her family were negative for food or contact allergy, angioedema, mastocytosis, or any systemic disorder, apart from psoriasis. Administration of acitretin was discontinued and $80 \mathrm{mg} /$ day methylprednisolone was intravenously given. Within 3 days the angioedema completely resolved. The results of further laboratory investigations including $\mathrm{C}_{1}$ esterase inhibitor, serum angiotensin-converting enzyme activity (SACE), hepatitis and HIV serology, serum complement, and parasitological investigations were either negative or normal. The patient refused any diagnostic procedures, except for the oral provocation test because it was the only one that could answer whether acitretin was the causative factor of angioedema.

One month later, re-exposure of the patient to oral acitretin resulted in a relapse of angioedema on the facial skin (without any urticarial lesions) that was successfully treated with intravenous administration of $80 \mathrm{mg} /$ day methylprednisolone. The patient was switched to other forms of antipsoriatic therapy and is presently completing a 38-month follow-up without any relapse of angioedema.

\section{Discussion}

Drug-induced angioedema associated with urticaria can be caused by IgE-mediated immunological mechanisms or by non-immunological mechanisms (5), whereas the pathogenesis of drug-induced angioedema without urticaria is far from being clearly understood. Moreover, because no underlying immunological mechanisms have been detected in the latter category of drug-induced angioedema, cutaneous allergy tests and determination of the serum 
levels of diverse antibodies cannot contribute to the identification of the causative drug (5). Drugs that are well known to be capable of inducing angioedema without urticaria include inhibitors of the angiotensin-converting enzyme, antagonists of angiotensin II type 1 receptor, fibrinolytic compounds, and nonsteroidal antiinflammatory drugs.

Angioedema has been previously reported in four patients treated with oral retinoids: isotretinoin (two cases without urticaria and two cases with associated urticaria) and in two patients treated with oral acitretin (one case with and one case without urticaria) (7-11).

To the best of our knowledge, the patient reported here is the second case of angioedema (without urticaria) caused by oral acitretin. The first case was reported by Filho et al. (8). Two days after acitretin initiation, the patient reported by these authors also revealed only periorbital swelling, whereas in our patient angioedema was diffuse on the facial skin, being more intense in the periorbital region and the lips. It seems that, in their case, discon-

\section{References}

1. Tsambaos D. Retinoids: a new chapter of systemic dermatotherapy. Dermatosen in Beruf und Umwelt. 1996;44:182-3.

2. Chroni E, Monastirli A, Tsambaos D. Neuromuscular adverse effects associated with systemic retinoid dermatotherapy: monitoring and treatment algorithm for clinicians. Drug Saf. 2010;33:25-34.

3. Misra L, Khurmi N, Trentman T. Angioedema: classification, management and emerging therapies for the perioperative physician. Indian J Anaesth. 2016;60:534-41.

4. Greaves MW, Lawlor F. Angioedema: manifestations and management. J Am Acad Dermatol. 1991;25:155-65.

5. Inomata N. Recent advances in drug-induced angioedema. Allergol Intern. 2012;61:545-57. tinuation of acitretin was enough to lead to a complete resolution of angioedema, whereas in our patient with a more severe clinical picture intravenous administration of steroids was necessary. The positive oral provocation tests performed in both cases clearly indicate that acitretin is capable of inducing angioedema (without urticaria). Theoretically, it is possible that not acitretin itself, but the ingredients of the retinoid-containing capsules (gelatin, red ferric oxide, yellow ferric oxide, sodium lauryl sulfate, and titanium dioxide), may be responsible for the occurrence of angioedema. However, this possibility seems very unlikely in view of the fact that, although these additives are widely used in the food and drug industry, they have not been found to cause angioedema (8).

Because oral acitretin is currently administered to numerous patients worldwide, it is of importance that dermatologists be fully aware of this potential adverse reaction and inform their patients to be treated with this compound that angioedema is a rare but serious side effect.

6. Lerch M. Drug-induced angioedema. Chem Immunol Allergy. 2012;97:98-105.

7. Saray Y, Seckin D. Angioedema and urticaria due to isotretinoin therapy. J Eur Acad Dermatol. 2006;20:118-20.

8. Filho RRDC, de Almeida HL Jr, Breunig JDA. Angioedema due to oral acitretin and isotretinoin. An Bras Dermatol. 2011;86:28-30.

9. Üstüner P. Angioedema due to systemic isotretinoin therapy. Case Rep Dermatol Med. 2014;2014:595914.

10. Zonunsanga N. Isotretinoin induced rash, urticaria and angioedema: a case report. Our Dermatol Online. 2015;4:460-2.

11. Solak B, Metin N, Erdem MT. Angioedema-urticaria due to acitretin. Am J Ther. 2016;23:e1116-7. 